\title{
A POSIÇÃO DO ALVO NA INFLUÊNCIA DO MOVIMENTO OCULAR EM TAREFAS DE PESQUISA NAVEGACIONAL E INFORMATIVA
}

\section{THE POSITION OF THE TARGET IN THE INFLUENCE OF EYE MOVEMENT ON NAVIGATIONAL AND INFORMATIVE RESEARCHTASKS}

\author{
J. Vasconcelos-Raposo, C. Teixeira, C. Alves, H. Lopes, M. Mendes, P. Andrade, M. Melo
}

ARTIGO ORIGINAL | ORIGINAL ARTICLE

O objetivo principal do deste estudo consiste em perceber de que forma a posição do alvo influencia o movimento ocular nas tarefas navegacional e informativa. O estudo integrou uma amostra de 20 estudantes universitários, 13 do sexo feminino e 7 do sexo masculino, com idades compreendidas entre os 18 e os 44 anos, de uma Universidade. Os participantes responderam a um questionário sociodemográfico e realizaram duas tarefas, uma navegacional e outra informativa. Foram realizadas combinações lineares, através do teste da MANOVA (2x2), de variáveis dependentes que maximizam as diferenças entre várias condições das variáveis independentes. Concluímos que a posição do alvo na tarefa navegacional influencia de forma significativa o movimento ocular dos estudantes universitários e a posição do individuo influencia o movimento ocular em ambas as tarefas, navegacional e informativa. Palavras-chave: movimento ocular, tarefa navegacional, tarefa informativa, posição do alvo, sexo

ABSTRACT

The main objective of this study is to understand how the position of the target influences the ocular movement in navigational and informative tasks. The study included a sample of 20 university students, 13 females and 7 males, aged between 18 and 44, from a university. Participants answered a socio-demographic questionnaire and performed two tasks, one navigational and one informative. Linear combinations were performed through the MANOVA test $(2 \times 2)$, of dependent variables that maximize the differences between several conditions of the independent variables. We conclude that the position of the target in the navigational task significantly influences the ocular movement of university students and the position of the individual influences the ocular movement in both tasks, navigational and informative.

Keywords: eye movement, navigational task, information task, target position, sex

Submetido: 11.17.2016 | Aceite: 03.24.2017

J. Vasconcelos-Raposo. Universidde de Trás-os-Montes e Alto Douro, INESC TEC., Health and Human Performance Lab. Vila Real. Portugal

C. M. Teixeira, C. Alves, H. Lopes, M. Mendes, P. Andrade. Universidade de Trás-os-Montes e Alto Douro, Dept Educação e Psicologia, Vila Real, Portugal.

M. Melo. MASSIVE-Lab, INESC TEC Pólo UTAD.

Endereço para correspondência: José Vasconcelos-Raposo. Universidde de Trás-os-Montes e Alto Douro, Q.ta de Prados, 5001801 Vila Real, Portugal

E-mail: jvraposo@utad.pt 
O movimento ocular tem sido usado em muitos domínios para captar a cognição visual, bem como o comportamento de interação dos utilizadores com computadores (Rayner, 1998; Jacob \& Karn, 2003). Os olhos nunca descansam numa posição por muito tempo. Eles movem-se várias vezes por segundo, com micro movimentos (Ehmke \& Wilson, 2007).

O movimento ocular permite registar a posição dos olhos enquanto eles se movem através de estímulos visuais. Assim, estes fornecem informações sobre a distribuição da atenção visual em termos dos objetos que são observados, pelo tempo e pela ordem. Isso é importante, já que foi sugerido que o que está a ser fixado pelos olhos indica o que está a ser processado na mente (Just \& Carpenter, 1980).

Estudar o movimento ocular durante atividades psicofísicas é importante para medir o controlo do movimento ocular obtendo informações, sobre um nível de comportamento que normalmente se encontra inacessível à introspeção consciente, estratégias de processamento de informações e também o desempenho de tarefas de controlo durante experiências que exigem fixação (Cornelissen, Petters, \& Palmer, 2002).

A fixação é um breve momento em que o olho está parado numa palavra e o cérebro processa a informação visual e varia entre os sujeitos (Ferreira, Schwieter, Gottardo, \& Jones, 2016) e uma deslocação é um movimento rápido do olho em que o olho se posiciona logo na secção seguinte do texto. Os indivíduos fazem muitos movimentos oculares quando estão perante grandes exibições (Beymer, Russell, \& Orton, 2008; Hooge \& Erkelens, 1998).

Com o Eye Tracking podem ser feitas análises para entender como as pessoas leem um texto. É feito através de uma câmara que grava para onde o olho do sujeito está a olhar, uma vez que esses pontos estão colocados no texto para seguir o comportamento dos sujeitos na leitura. O olho move-se durante o processo de leitura e lê uma linha de texto fazendo uma série de fixações e deslocações. Quando olhamos para alguma coisa, lemos ou procuramos algum objeto, fazemos os chamados movimentos oculares "sacádicos" (Bertera \& Rayner, 2000).

No que diz respeito a tarefas na web, a pesquisa é uma das ações mais comuns do nosso quotidiano e quanto menor for o tempo necessário para encontrar o alvo, mais eficaz esta é. Tendo como referência os padrões do movimento ocular e para os estímulos mais simples, o alvo é encontrado com um pequeno número de fixações, mas as durações médias destas são altas. Durante a pesquisa na web, o número de vezes que os utilizadores olham para o alvo depois da primeira fixação fornece informações acerca do desenvolvimento do processo de pesquisa (Bojko, 2006). À medida que o grau de complexidade do estímulo aumenta, o número de fixações necessárias para encontrar o estímulo alvo aumenta, e a duração média das fixações diminui (De Macedo, Covre, Orsati, De Oliveira, Schwartzman, 2007). Segundo De Macedo et al., (2007) a análise dos movimentos oculares pode também sugerir diferenças no processamento ocular nas tarefas fáceis e difíceis. No entanto, há que ter em conta que o facto de conhecer o objetivo da tarefa acelera a deteção do alvo em tarefas de pesquisa na web. Isso indica que o nosso sistema visual prejudica o nosso sistema atencional, pois a apresentação antecipada do alvo faz com que o objetivo seja mais fácil de realizar (Navalpakkam \& Itti, 2005). No que diz respeito ao alvo, um estudo realizado por Greene e Rayner (2001) aponta que quando o alvo é desconhecido os sujeitos encontram-no facilmente. No entanto, quando o indivíduo não está familiarizado com as distrações é menos eficiente a encontrar o alvo.

Com base nas características dos dados de Eye Tracking, como por exemplo a duração da fixação e o tamanho da pupila, realizaram-se vários estudos. Num desses, os sujeitos dispu- 
nham de uma lista de resultados e a partir dela selecionavam links para consulta. Concluiu-se que os sujeitos passam muito tempo a fixar o primeiro e o segundo resultado antes de selecionar um link (Granka, Joachims, \& Geri, 2004). Pesquisas indicam que, a atração do site tem um poderoso impacto na perceção que o sujeito tem da página web (Lavie \& Tractinsky, 2004; Lindgaard, Fernandes, Dudek, \& Browñ, 2006). Neste sentido, o sujeito é orientado por uma necessidade de informação, ou seja, a intenção do indivíduo para realizar uma pesquisa na web muitas vezes não é informativa, mas sim de navegação (obter o URL de um site desejado) ou pode ser transacional (o indivíduo tem como objetivo executar uma determinada transação, como por exemplo, comprar ou descarregar um documento) (Broder, 2012).

Num outro estudo, os utilizadores tinham expectativas claras de onde os objetos na web estavam localizados. No entanto, a disponibilidade de objetos na web levou a menos fixações e os participantes encontraram os objetos mais rapidamente (Roth, Tuch, Mekler, Bargas-Avilan \& Opwis, 2013). Num estudo sobre tarefas de pesquisa, os participantes navegaram livremente em várias páginas da web, enquanto realizavam tarefas específicas, como a remoção de links. A navegação entre colunas mostrou-nos que os participantes são ligeiramente mais propensos a mudar de visão entre as colunas do que ficar fixado dentro de uma coluna (Goldberg, Stimson, Lewenstein, Scott, \& Wichansky, 2002).

No que diz respeito a anúncios presentes em páginas web, as evidências sugerem que os movimentos oculares estão fortemente relacionados com a ordem em que os anúncios estão apresentados. O tipo de tarefa, os anúncios e a sequência influenciam a pesquisa (Buscher, Dumais, \& Cutrell, 2010). Estes podem ser usados para representar o processamento da informação (Underwood \& Radach, 1998).

Tendo como referência estudos que consi- deraram as características do utilizador, o padrão de fixações em quase todas as páginas da web mostrou que os adultos olhavam mais tempo para partes da página do que os jovens adultos (Wass, Smith, \& Johnson, 2013).

O propósito do presente trabalho consiste em perceber de que forma a posição do alvo nas tarefas navegacional e informativa influência o movimento ocular, em estudantes universitários. Pretendendo assim comparar a relação entre a posição do alvo nas tarefas navegacional e informativa com a velocidade do movimento ocular assim como, a relação entre a posição do indivíduo com o movimento ocular.

\section{MÉTODO}

Este é um estudo quasi-experimental, transversal de caráter quantitativo.

\section{Amostra}

Vinte participantes, 13 mulheres e 7 homens com idades compreendidas entre os 18 e os 44 anos foram utilizados para este estudo. Os níveis de experiência na utilização da web evidenciaram que $75 \%$ dos participantes usa diariamente a web. $35 \%$ dos indivíduos passa entre 2-3 horas por dia na web, $30 \%$ passa entre 3-4 horas por dia e 35\% passa mais de 5 horas por dia na web. $75 \%$ dos participantes não usam óculos e dessa percentagem apenas $33.3 \%$ são do sexo masculino. $50 \%$ dos participantes já utilizaram óculos de realidade virtual.

\section{Instrumentos}

Os dados do movimento ocular ajudam-nos a perceber onde as pessoas focam a atenção e de que forma fazem a seleção. Nesse sentido, foi utilizado um Eye tracker para registar para onde as pessoas olhavam durante a pesquisa. O Eye Tracker é um equipamento que permite fazer o rastreamento dos movimentos oculares e recolher informações sobre o processamento de informações visuais. Este equipamento tem sido utilizado para pesquisas de informações em páginas 
da web (Aula, Majaranta, \& Räihä, 2005).

Como estímulos, foram utilizados o site do youtube para a tarefa navegacional e momondo para a tarefa informativa recorremos a um site de promoção, vendas e reserva de viagens.

\section{Procedimentos}

Tendo em consideração que os objetivos específicos visam comparar grupos e que as variáveis independentes são três (tipo de tarefa (navegacional e informativa), posição do alvo e posição do indivíduo) e que as variáveis dependentes são três (fixação média, número de piscadelas e duração média de piscadelas) recorremos à MANOVA $(2 \times 2)$, uma vez que se verificou a existência de uma distribuição normal.

O nível de significância foi mantido em $5 \%$ $(p<.05)$.

\section{RESULTADOS}

Foram realizadas combinações lineares de variáveis dependentes que maximizam as diferenças entre várias condições das variáveis independentes. Antes de proceder à análise muiltivariada, foi assegurada a distribuição normal dos dados através da Assimetria e Curtose $( \pm 2)$.

As variáveis independentes apresentaram um efeito forte: Sexo $\times$ Posição do alvo na tarefa informativa $\left(\mathrm{F}_{(6,2)}=.483, p=.793\right.$, $\eta_{\mathrm{p}}^{2}=.592$, PO $=.073$, Wilks $\left.\lambda=.408\right)$; Sexo $\times$ Posição do indivíduo $\left(\mathrm{F}_{(6,2)}=.398, p=.839\right.$, $\eta_{\mathrm{p}}^{2}=.544, \mathrm{PO}=.069$, Wilks $\left.\lambda=.456\right)$.

De acordo com os resultados apresentados na Quadro 1, verificou-se que existe um efeito forte entre a combinação de variáveis Fixação média na tarefa navegacional $\times$ Posição do alvo na tarefa navegacional $\left(n_{\mathrm{p}}^{2}=.167\right)$.

Quadro 1

Posição do alvo e movimento ocular nas tarefas navegacional e informativa

\begin{tabular}{|c|c|c|c|c|}
\hline Variáveis & $\mathrm{F}$ & $p$ & $\eta_{p}{ }^{2}$ & $\mathrm{PO}$ \\
\hline $\begin{array}{l}\text { Fixação média na tarefa navegacional } \times \text { Posição do alvo na } \\
\text { tarefa navegacional }\end{array}$ & 0.734 & 0.553 & 0.167 & 0.159 \\
\hline $\begin{array}{l}\text { Fixação média na tarefa informativa } \times \text { Posição do alvo na } \\
\text { tarefa informativa }\end{array}$ & 0.448 & 0.517 & 0.039 & 0.940 \\
\hline
\end{tabular}

Por outro lado, existe um efeito moderado na combinação de variáveis Fixação média na tarefa informativa $\times$ Posição do alvo na tarefa informativa $\left(n_{\mathrm{p}}^{2}=.039\right)$. Foram, ainda, obtidos os seguintes resultados para a variável dependente, fixação média na tarefa navegacional (255.410 - 326.931) e para a variável fixação média na tarefa informativa (274.031 - 374.527). Como se pode observar a partir das comparações entre as diferentes posições do alvo, podemos verificar que existe um efeito moderado entre duas combinações de variáveis: Posição do alvo na tarefa navegacional e a Duração média de piscadelas na tarefa navegacional $\left(n_{\mathrm{p}}^{2}=.093\right)$; Posição do alvo na tarefa informativa $\times$ Duração média de piscadelas na tarefa informativa $\left(\eta_{\mathrm{p}}^{2}=.085\right)$. Já o número de piscadelas $\times$ Posição do alvo na tarefa navegacional apresenta um efeito forte $\left(\eta_{\mathrm{p}}^{2}=.192\right)$.

Pelo contrário, a combinação Posição do alvo na tarefa informativa $\times$ Duração média de fixações e a combinação Posição do alvo na tarefa informativa $\times$ Número de piscadelas apresentam um efeito moderado: $\eta_{\mathrm{p}}{ }^{2}=.085 \mathrm{e}$ $\eta_{\mathrm{p}}^{2}=.013$ respetivamente.

A duração média de piscadelas na tarefa navegacional (204.236 - 1656.397). A variável duração média de piscadelas na tarefa informativa (553.598 - 1864.440). A variável número de piscadelas na tarefa navegacional (1.987 9.786) e por último, a variável número de piscadelas na tarefa informativa (9.648 - 20.040).

De acordo com os resultados relativos à posição dos individuos, foi possível verificar que existe um efeito forte entre as combinações de variáveis Fixação média na tarefa nave- 
gacional $\times$ Posição do indivíduo $\left(\eta_{\mathrm{p}}^{2}=.216\right) \mathrm{e}$ Fixação média na tarefa informativa $\times$ Posição do indivíduo $\left(\eta_{\mathrm{p}}^{2}=.176\right)$.
A variável dependente, fixação média na tarefa navegacional (263.566 - 322.430). A variável fixação média na tarefa informativa (270.076 - 342.473).

Quadro 2

Posição do alvo e rapidez do movimento ocular nas tarefas navegacional e informativa

\begin{tabular}{|c|c|c|c|c|}
\hline Variáveis & $F$ & $p$ & $\eta_{p}^{2}$ & $P O$ \\
\hline $\begin{array}{l}\text { Posição do alvo na tarefa navegacional } \times \text { Duração média de } \\
\text { piscadelas na tarefa navegacional }\end{array}$ & 0.377 & 0.772 & 0.093 & 0.102 \\
\hline $\begin{array}{l}\text { Posição do alvo na tarefa navegacional } \times \text { Número de piscadelas } \\
\text { na tarefa navegacional }\end{array}$ & 0.869 & 0.486 & 0.192 & 0.181 \\
\hline $\begin{array}{l}\text { Posição do alvo na tarefa informativa } \times \text { Duração média de } \\
\text { piscadelas na tarefa informativa }\end{array}$ & 1.018 & 0.335 & 0.085 & 0.152 \\
\hline $\begin{array}{l}\text { Posição do alvo na tarefa informativa } \times \text { Número de piscadelas } \\
\text { na tarefa informativa }\end{array}$ & 0.013 & 0.912 & 0.013 & 0.051 \\
\hline
\end{tabular}

Quadro 3

Posição do indivíduo e movimento ocular nas tarefas navegacional e informativa

\begin{tabular}{lcccc}
\hline Variáveis & $F$ & $p$ & $\eta_{\mathrm{p}}{ }^{2}$ & $P O$ \\
\hline Fixação média na tarefa naveg $\times$ Posição do individuo & 4.398 & 0.572 & 0.216 & 0.504 \\
Fixação média na tarefa informativa $\times$ Posição do individuo & 3.411 & 0.083 & 0.176 & 0.412 \\
\hline
\end{tabular}

\section{DISCUSSÃO}

Relativamente ao nosso primeiro propósito que consiste em perceber se a posição do alvo, nas tarefas informativa e navegacional, influencia o movimento ocular em estudantes universitários, a literatura sugere que os olhos do indivíduo nunca permanecem fixados numa determinada posição durante muito tempo, isso deve-se ao facto de estarem fortemente relacionados com a ordem em que os anúncios estão apresentados, isto é, o tipo de tarefa influencia a pesquisa (Just \& Carpenter, 1980; Ehmke \& Wilson, 2007; Buscher, Dumais \& Cutrell, 2010).

De acordo com o segundo objetivo deste estudo, comparar a relação entre a posição do alvo em ambas as tarefas com a rapidez do movimento ocular a literatura diz-nos que a análise da duração dos movimentos oculares difere dependendo das tarefas realizadas. Neste caso em particular, quando o alvo era de tipo navegacional o movimento ocular foi correlacionado mais fortemente ao contrário do tipo informativo (De Macedo et al., 2007). Estes resultados podem ser explicados pelo facto de os estudantes universitários estarem mais familiarizados com a tarefa navegacional do que com a tarefa informativa. Assim, os resultados obtidos corroboram a literatura.

$\mathrm{O}$ facto de na tarefa navegacional apresentar maiores níveis de movimento ocular pode ser explicado pelo facto do site de pesquisa navegacional utilizado no nosso estudo (youtube) ser familiar a todos os participantes, e resultar 
numa pesquisa mais breve e rápida relativamente com o site de pesquisa informativa.

Sumariamente, a posição do indivíduo influenciou o movimento ocular dos participantes. Assim, o facto de o indivíduo estar sentado ou de pé influencia o tipo de tarefa que esta a realizar.

As limitações do nosso estudo consitem no facto de a amostra ser de dimensão reduzida e que o número de sujeitos por sexo também está desiquilibrado. Futuramente, seria pertinente a realização do mesmo estudo com uma amostra mais alargada, com um número aproximado de participantes do sexo masculino e feminino. Também deverá ser realizada o mesmo tipo de tarefa, navegacional e informativa com vários links. Também poderá ser adequado realizar estudos posteriores para verificar se das posições do individuo (sentado ou de pé) influencia de forma significativa o movimento ocular.

\section{CONCLUSÕES}

Podemos concluir que a posição do alvo na tarefa navegacional influencia o movimento ocular dos estudantes universitários.

Em suma, os estudantes ao realizarem a tarefa navegacional apresentaram maiores níveis de movimento ocular comparativamente com o tipo de tarefa informativa.

Por outro lado, a posição do indivíduo também influencia o movimento ocular em ambas as tarefas, navegacional e informativa..

Os participantes revelaram uma maior rapidez no movimento ocular no site de pesquisa navegacional, uma vez que os anúncios que são apresentados em cada página vão influenciar o tipo de movimentação ocular, visto que, o site da tarefa de pesquisa navegacional, para além de ser mais comum aos participantes era mais organizado ao nível dos estímulos visuais. O site navegacional é organizado em coluna o que facilita a mudança de olhar entre estas.
Agradecimentos:

Nada declarado.

\section{Conflito de Interesses:}

Nada declarado.

\section{Financiamento:}

Nada declarado.

\section{REFERÊNCIAS}

Aula, A., Majaranta, P., \& Räihä, K. (2005). Eye-tracking reveals the personal styles for search result evaluation. Interact, 1058-1061.

Bertera J. H., \& Rayner K. (2000). Eye movements and the span of the effective stimulus in visual search. Percept Psychophys, 62(3), 576-85.

Beymer, D., Russell, D., \& Orton, P. Z. (2008). An eye tracking study of how font size, font type, and pictures influence online reading. The Author, 456-460.

Bojko, A. (2006). Using eye tracking to compare web page designs: A case study. Journal of Usability Studies, 1(3), 112-120.

Broder, A. (2002). A taxonomy of web search. ACM Sigir fórum, 36(2), 3-10.

Buscher, G., Dumais, S. T., \& Cutrell, E. (2010). The good, the bad, and the random: an eye-tracking study of ad quality in web search. In Proceedings of the 33rd international ACM SIGIR conference on Research and development in information retrieval. 42-49.

Cornelissen, F. W., Peters, E. M., \& Palmer, J. (2002). The Eyelink Toolbox: eye tracking with MATLAB and the Psychophysics Toolbox. Behavior Research Methods, Instruments, \& Computers, 34(4), 613-617.

De Macedo, E. C. D., Covre, P., Orsati, F. T., Okada, M., \& Schwartzman, J. S. (2007). Análise dos padrões dos movimentos oculares em tarefas de busca visual: efeito 
da familiaridade e das características físicas do estímulo. Arquivo Brasileiro Oftalmologico, 70(1), 31-6.

Ehmke, C., \& Wilson, S. (2007). Identifying web usability problems from eye-tracking data. In Proceedings of the 21st British HCI Group Annual Conference on People and Computers: HCI 1. 119-128.

Ferreira, A., Schwieter, J. W., Gottardo, A., \& Jones, J. (2016). Cognitive effort in direct and inverse translation performance: Insight from eye-tracking technology. Cadernos de Tradução, 36(3), 60-80. doi. org/10.5007/2175-7968.2016v36n3p60

Goldberg, J.H., Stimson, M.J., Lewenstein, M., Scott, N \& Wichansky, A.M. (2002). Eye tracking in web search tasks: design implications. In Proceedings of the 2002 symposium on Eye Tracking Research \& Applications. ACM. 51-58.

Granka, L. A., Joachims, T., \& Geri, G. (2004). Eye-tracking analysis of user behavior in WWW search. SIGIR, 478-479.

Greene, H. H., \& Rayner, K. (2001). Eye movements and familiarity effects in visual search. Vision Research, 41(27), 3763-3773. doi.org/10.1016/S0042 $-6989(01) 00154-7$

Hooge, I. T. C., \& Erkelens, C. J. (1998). Adjustment of fixation duration in visual search. Vision research, 38(9), 1295-IN4. doi.org/10.1016/S0042-6989(97)00287-3

Jacob, R. J. K., \& Karn, K. S. (2003). Comentary on section 4: Eye tracking in humancomputer interaction and usability research: ready to deliver the promises. Mind's Eye, 573-605. doi.org/10.1016/ B978-044451020-4/50031-1

Just, M. A., \& Carpenter, P. A. (1980). A theory of reading: From eye fixations to comprehension. Psychological Review, 87,
329-355.

Lavie, T., \& Tractinsky, N. (2004). Assessing dimensions of perceived visual aesthetics of web sites. International Journal of Human-Computer Studies, 60(3), 269-298. doi:10.1016/j.ijhcs.2003.09.002

Lindgaard, G., Fernandes, G., Dudek, C., \& Browñ, J. (2006). Attention web designers: You have 50 milliseconds to make a good first impression. Behaviour \& Information Technology, 25(2), 115-126. DOI: $10.1080 / 01449290500330448$

Navalpakkam, V., \& Itti, L. (2005). Modeling the influence of task on attention. Vision Research, 45(2), 205-231. DOI: 10.1016/j. vires.2004.07.042

Rayner, K. (1998). Eye movements and information processing: 20 years of research. Psychological Bulletin, 124(3), 372-422.

Roth, S. P., Tuch, A. N., Mekler, E. D., Bargas-Avila, J. A., \& Opwis, K. (2013). Location matters, especially for non-salient features - An eye-tracking study on the effects of web object placement on different types of websites. International Journal of Human-Computer Studies, 71(3), 228-235. doi. org/10.1016/j.ijhcs.2012.09.001

Underwood, G., \& Radach, R. (1998). Eye guidance and visual information processing: Reading, visual search, picture perception and driving. In G.Underwood (Ed.), Eye guidance in reading and scene perception (pp. 1-28). Oxford, England: Elsevier Science Ltd.

Wass, S.V., Smith, T.J. \& Johnson, M.H. (2013). Parsing eye-tracking data of variable quality to provide accurate fixation duration estimates in infants and adults. Behavior Research Methods, 45 (1), 229-250. Doi:10.3758/s13428-012-0245-6 